\title{
An Integrated Workflow To Investigate Electrocatalytic Surfaces By Correlative X- ray Photoemission Spectroscopy, Scanning Photoemission Electron Microscopy and Atom Probe Tomography
}

Kevin Schweinar ${ }^{1 *}$, Olga Kasian ${ }^{2}$, Rachel L. Nicholls ${ }^{3}$, Catherine R. Rajamathi ${ }^{3}$, Patrick Zeller ${ }^{4}$, Matteo Amati $^{4}$, Luca Gregoratti ${ }^{4}$, Dierk Raabe ${ }^{1}$, Mark Greiner ${ }^{3}$ and Baptiste Gault ${ }^{1,5}$

1. Max-Planck-Institut für Eisenforschung GmbH, Microstructure Physics and Alloy Design, Düsseldorf, Germany

2. Max-Planck-Institut für Eisenforschung GmbH, Interface Chemistry and Surface Engineering, Düsseldorf, Germany

3. Max-Planck-Institut für Chemische Energiekonversion, Heterogeneous Reactions, Mülheim a.d. Ruhr, Germany

4. Elettra - Sincrotrone Trieste S.C.p.A., Trieste, Italy

5. Imperial College, Royal School of Mines, Department of Materials, London, SW7 2AZ, UK

*Corresponding author: k.schweinar@mpie.de

The increasing share of renewable electricity in the energy mix requires the development of sustainable technologies capable of buffering intermittent energy provided by renewable sources. Proton exchange membrane water electrolysis (PEMWE) is seen as a promising technology to generate hydrogen as intermediate energy carrier between energy production and demand for end-use. The requirements for catalyst materials used in such applications are very demanding as they must have a high electronic conductivity, activity and long-term stability in harsh electrochemical environments. X-ray photoemission spectroscopy (XPS) and atom probe tomography (APT) is considered a suitable combination of methods to study changes in the electronic state and corresponding surface chemistry. Yet, conventional XPS averages over large areas, which does not allow for the detection of localized surface heterogeneities that may strongly affect the catalyst's performance [1]. Spatially-resolved measurements are required. Here, we propose an integrated workflow for the spatially correlated investigation of electrocatalysts combining electrochemical measurements, spatially-resolved scanning photoemission electron microscopy (SPEM) and APT performed on the same specimen.

Proof-of-concept experiments were conducted on $\mathrm{IrRu}$ thin films that are considered as promising candidates for the anodic oxygen evolution reaction (OER) in PEMWE devices. The samples were sputter-deposited on commercially available silicon microtip arrays, featuring 36 cone frusta with a diameter of $3 \mu \mathrm{m}$ at the top (Fig. 1a and b). The top surfaces provide an ideal area that is accessible for SPEM and subsequent correlated APT measurements. One sample was electrochemically oxidized by anodic polarization in a deuterated acidic solution prior to spectroscopy. Isotopic labelling was used to estimate the degree of hydroxylation of the electrochemical oxide with APT. Samples were then transferred into a vacuum container and constantly kept within a protective atmosphere during transport and mounting into the SPEM. The employed SPEM instrument utilizes zone plate optics to focus the incident photon beam to a spot of about $130 \mathrm{~nm}$, as described elsewhere [3]. This allows for (i) spectroimaging of the flat top area of the tips to detect possible lateral heterogeneities and (ii) collection of XP spectra from desired areas of the imaged surface (Fig. 1c). After recording the spectra, the samples remained in a controlled inert atmosphere at all times, and were transferred into a physical vapor deposition setup equipped with an e-beam evaporator. The samples were then coated with $200 \mathrm{~nm}$ of $\mathrm{Cr}$ to protect the top surface from further exposure to air. Following this, individual tips were sharpened to 
needle-shaped APT specimen using a dual-beam FIB-SEM. Care was taken that the interface $\mathrm{Cr}$-IrRu was eventually located close to the apex of the APT needle. This enabled the measurement of the very top surface layers of the catalyst thin film which were originally exposed to the electrochemical environment and investigated with spectroscopy (Fig. 1d and e). The final APT measurement was performed on a LEAP 5000XR instrument in laser pulsing mode.

This work demonstrates a well-advanced experimental strategy to investigate electrochemical surfaces. While the electrochemical data of a sample yields information on the catalyst's performance, complementary SPEM reveals the chemical state of the surface. Determined metal oxidation states can be correlated to oxygen contents determined by APT. Further, segregation phenomena along microstructural defects and leaching of an alloying component during OER can be investigated. This work can be expanded to different kind of thin film materials in the future and will help establishing structure-function relationships of catalytic materials [4].

[1] T Li et al, Nat. Catalysis 1 (2018), p. 300-305

[2] O Kasian et al, J. Electrochem. Soc. 163 (2016), F3099-F3104

[3] M Amati et al, Surf. Science 652 (2016), p. 20-25

[4] The authors wish to thank Elettra Sincrotrone for the allocation of beamtime at the ESCAmicroscopy beamline. The research leading to these results has received funding from the European Community's Horizon 2020 Framework Programme under grant agreement no 730872 . K.S. acknowledges IMPRSSurMat for funding. U. Tezins and A. Sturm are acknowledged for their technical support at MPIE.
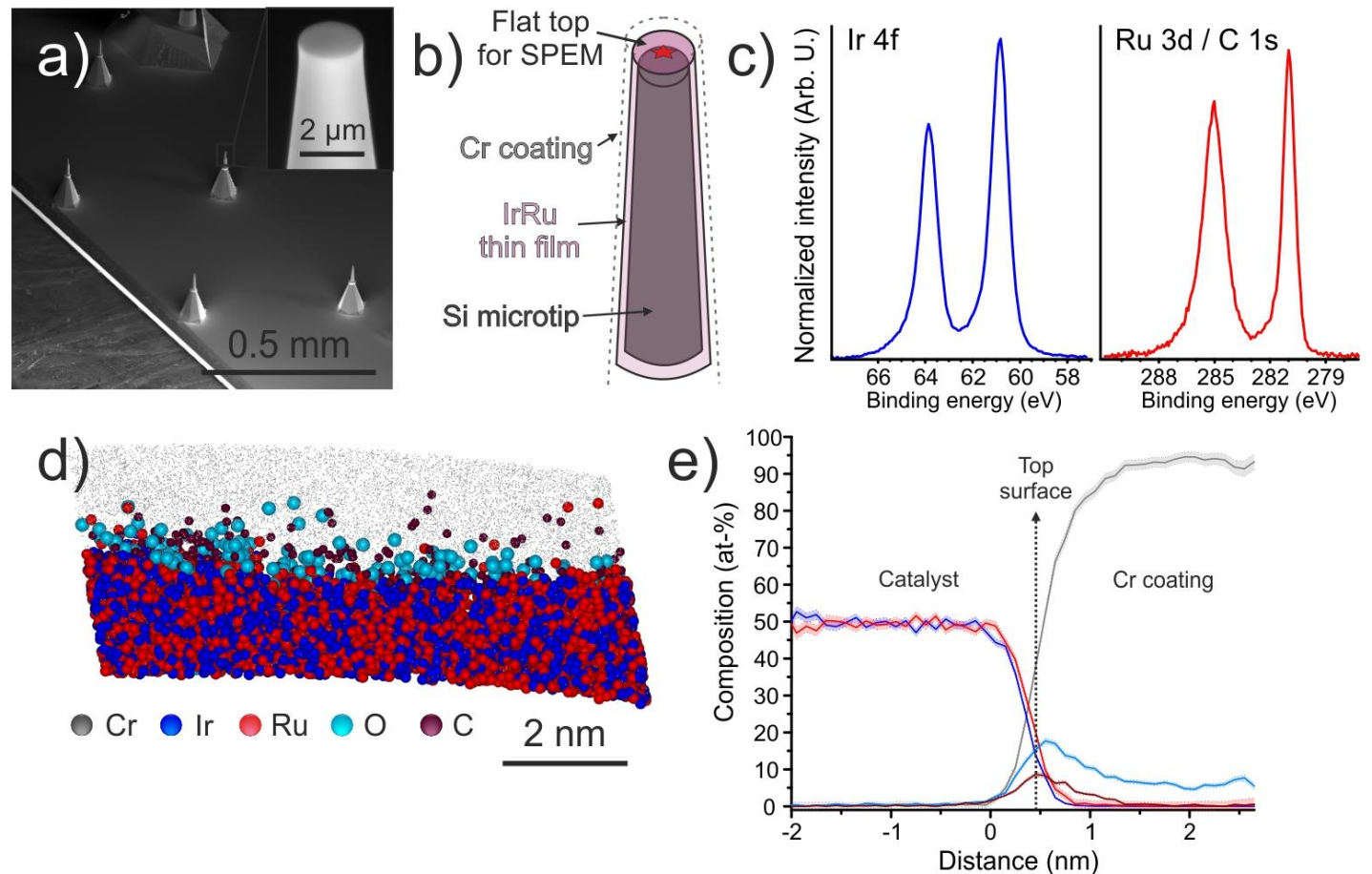

Figure 1. a) SEM overview image of Si microtip array; inset shows a zoom-in on a single flat top microtip. b) Schematic drawing of the final microtip sample design for correlative SPEM and APT before FIB sharpening. c) XP core-level spectra of Ir 4f and Ru 3d levels measured on a flat top of a microtip. d) Exemplary APT reconstruction and e) corresponding compositional profile of an asdeposited $\operatorname{Ir}_{50} \mathrm{Ru}_{50}$ thin film. Corresponding XP spectra of this area are shown in $\mathrm{c}$ ). 